\title{
ZERO-ONE LAWS FOR GAUSSIAN MEASURES ON BANACH SPACE(1)
}

BY

\section{CHARLES R. BAKER}

\begin{abstract}
Let $B$ be a real separable Banach space, $\mu$ a Gaussian measure on the Borel $\sigma$-field of $\$$, and $B_{\mu}[B]$ the completion of the Borel $\sigma$-field

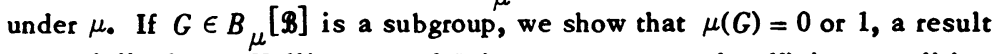
essentially due to Kallianpur and Jain. Necessary and sufficient conditions are given for $\mu(G)=1$ for the case where $G$ is the range of a bounded linear operator. These results are then applied to obtain a number of $0-1$ statements for the sample function properties of a Gaussian stochastic process. The zero-one law is then extended to a class of non-Gaussian measures, and applications are given to some non-Gaussian stochastic processes.
\end{abstract}

1. Introduction. Kallianpur [12] has proved the following result. Let $T$ be a complete separable metric space, $\chi$ a linear space of real-valued functions on $T$, and $B[\chi]$ the $\sigma$-field of $\chi$ sets generated by sets of the form $\left\{x:\left(x\left(t_{1}\right), \cdots\right.\right.$, $\left.\left.x\left(t_{n}\right)\right) \in C\right\}, t_{1}, \cdots, t_{n} \in T$ and $C$ a Borel set in $R^{n}$. Suppose that $P$ is a Gaussian probability measure on $B[\chi]$ with continuous covariance function $K$ and zero mean, and that $\chi$ contains the reproducing kernel Hilbert space of $K$. Let $B_{0}[\chi]$ denote the completion of $B[\chi]$ under $P$. With these assumptions, Kallianpur has shown that $P(G)=0$ or 1 for every $B_{0}[\chi]$-measurable $r$-module of $\chi$. This result was extended to subgroups by Jain [11]. Moreover, an inspection of the proofs in [12] and [11] reveals that the zero-one law holds for Gaussian measures with nonzero mean, after making the necessary change in the form of the Radon-Nikodym derivative of a Gaussian measure equivalent to $P$.

We first show that this zero-one law holds for Gaussian measures on a real separable Banach space. Necessary and sufficient conditions for the alternatives are also given for cases where the subgroup is the range space of a bounded linear operator. A number of applications are given on path properties of Gaussian stochastic processes. Finally, the zero-one law is extended to a class of non-

Received by the editors December 14, 1971. $60 \mathrm{G} 30$.

AMS (MOS) subject classifications (1970). Primary 28A40, 60G15, 60G17; Secondary

Key words and phrases. Gaussian measures, Gaussian stochastic processes, zeroone laws.

(1) This research was supported in part by the Air Force Office of Scientific Research under Contract AFOSR-68-1415 and by the Office of Naval Research under Contract N00014-67-A-0;21-0006 (NR 042-269). 
Gaussian measures, and some zero-one laws are given for a class of nonGaussian stochastic processes.

As noted in [12], Cameron and Graves [2] first considered this problem for the case of Wiener measure. In another direction, Pitcher [18] essentially proved the following result. Let $\mu$ be a zero-mean Gaussian measure on a real separable Hilbert space $\mathcal{H}$ with covariance operator $K$. Suppose $K=T S T^{*}$ for $S$ and $T$ Hilbert-Schmidt linear operators. Then $\mu[\mathrm{range}(T)]=0$ or 1 . Our interest in this problem was motivated by Pitcher's work. Results similar to those of Kallianpur, but restricted to linear manifolds, have been obtained by Rosanov [19].

2. Definitions. $B$ will denote a separable Banach space with norm $\|\cdot\|, \mathcal{H}$ a separable Hilbert space with inner product $\langle\cdot, \cdot\rangle$. All linear spaces are defined over the real numbers. For a given topological space $\mathfrak{Q}, B[\mathfrak{Q}]$ will denote the Borel $\sigma$-field of $\mathfrak{Q}$ sets. If $\mu$ is a probability measure on $B[\mathfrak{Q}]$, then $B_{\mu}[\mathfrak{Q}]$ will denote the completion of $B[\mathfrak{Q}]$ under $\mu$. A covariance operator in $\mathcal{H}$ is an operator that is bounded, linear, nonnegative, selfadjoint, and trace-class.

A Gaussian measure $\mu$ on $B[\mathfrak{B}]$ is by definition a probability measure such that all bounded linear functionals on $\mathfrak{B}$ are Gaussian with respect to $\mu$. $\mathfrak{B}^{*}$ is the space of all bounded linear functionals on $\mathfrak{B}$.

3. The zero-one law for Banach space. In this section, we obtain the zeroone law for Gaussian measures on a separable Banach space. To obtain this result, we will first show that Kallianpur's result, and Jain's extension, includes Gaussian measures on a separable Hilbert space.

We recall that if $\mu$ is a Gaussian measure on $B[\mathcal{H}]$, then $\mu$ has a mean element $m$ and covariance operator $R$, defined by

$$
\langle m, y\rangle=\int_{\mathcal{H}}\langle x, y\rangle d \mu(x), \quad\langle R u, y\rangle=\int_{\mathcal{H}}\langle x-m, u\rangle\langle x-m, y\rangle d \mu(x)
$$

for all $u, y$ in $\mathcal{H}[16]$.

Lemma 1. Let $\mu$ be a Gaussian measure on $B[\mathcal{H}]$, and suppose that $G$ is a subgroup of $\mathcal{H}, G \in B_{\mu}[\mathcal{H}]$. Then $\mu(G)=0$ or 1 .

Proof. Let $\mathcal{R}$ denote the function on $\mathcal{H} \times \mathcal{H}$ defined by $\mathcal{R}(u, v)=\langle R u, v\rangle$, where $R$ is the covariance operator of $\mu$. Let $W: \mathcal{H} \rightarrow \mathcal{H}^{*}$ be given by $W u^{\prime}=u$ when $u(x)=\left\langle u^{\prime}, x\right\rangle$ for all $x \in \mathcal{H} . W$ is continuous, linear, one-to-one and onto. It is straightforward to show that the reproducing kernel Hilbert space $H(R)$ of $R$ is equal to $W\left[\right.$ range $\left.\left(R^{1 / 2}\right)\right]$ with the inner product $\langle u, v\rangle_{H(R)}=\left\langle x_{u}, x_{v}\right\rangle$, where $x_{u}$ is the unique element of range(R) satisfying $W R^{1 / 2} x_{u}=u$. Now let $\nu$ be the Gaussian measure on $B\left[\mathcal{H}^{*}\right]$ defined by $\nu[A]=\mu\left[W^{-1}(A)\right]$. The covariance function of $\nu$ is $\mathscr{R} ; \mathfrak{R}$ is continuous on $\mathcal{H} \times \mathcal{H}$, and $H(R) \subset \mathcal{H}^{*}$. We can thus apply 
the $0-1$ law of [12] and [11] to $\nu$. Since $W$ is linear, $W[G]$ is a subgroup; since $W^{-1}$ is continuous and one-to-one, $W[G]$ belongs to $B_{\nu}\left[\mathcal{H}^{*}\right]$. Hence $\nu[W(G)]=$ $\mu[G]=0$ or 1 .

Now let $\mu$ be a Gaussian measure on $B[\Re]$ and suppose that $G \in B_{\mu}[\Re]$ is a subgroup of $\mathfrak{B}$.

Theorem 1. $\mu(G)=0$ or 1 .

Proof. We can construct a separable Hilbert space $\mathcal{H}$ such that the elements of $\mathfrak{B}$ constitute a dense linear manifold in $\mathcal{H}, \mathfrak{B} \in B[\mathcal{H}]$, and $B[\mathfrak{B}]=\mathscr{B} \cap B[\mathcal{H}]$; see [14]. Let $\nu$ be the Gaussian measure on $B[\mathcal{H}]$ defined by $\nu(A)=\mu(\mathfrak{B} \cap A)$. Let $B_{\nu}[\mathcal{H}]$ be the completion of $B[\mathcal{H}]$ under $\nu$. Since all sets in $B[\Re]$ of $\mu$-measure zero belong to $B[\mathcal{H}]$ and have $\nu$-measure zero, it is clear that $B \nu[\mathcal{H}]$ contains all elements of $B_{\mu}[\mathfrak{B}]$. Hence, if $G$ is a subgroup in $B_{i} G \in B_{\mu}[\mathfrak{B}]$, then $G$ is a subgroup in $\mathcal{H}, G \in B_{\nu}[\mathcal{H}]$. The result now follows from Lemma 1 and the definition of $\nu$.

4. Measurable subgroups. The following two lemmas can often be used to show that a given subgroup is measurable.

Lemma 2 [17]. Let $T$ be a map from a complete separable metric space $\pi_{1}$ into a complete separable metric space $\pi_{2}$. Suppose $T$ is one-to-one and $B\left[\pi_{1}\right] / B\left[\pi_{2}\right]$ measurable. Then $T[A] \in B\left[\pi_{2}\right]$ when $A \in B\left[\pi_{1}\right]$.

In our applications, we deal with a bounded linear operator $T$ between two Banach spaces. The following lemma extends Lemma 2 (Kuratowski's theorem) to the case where $\pi_{T}$ (the null space of $T$ ) contains elements other than the null element.

Lemma 3. Let $\mathscr{B}_{1}$ and $\mathscr{B}_{2}$ be two separable Banach spaces, with $T: \mathscr{B}_{1} \rightarrow$ $\mathfrak{B}_{2}$ a bounded linear operator. Then

(1) $B_{1}$ and $\overline{\text { range }(T)}$ can be imbedded as measurable dense linear manifolds in Hilbert spaces $\mathcal{H}_{1}$ and $\mathcal{H}_{2}$ so that $B\left[\mathscr{B}_{1}\right]=\mathscr{B}_{1} \cap B\left[\mathcal{H}_{1}\right], B[\overline{\operatorname{range}(T)}]=$ $\overline{\text { range }(T)} \cap B\left[\mathcal{H}_{2}\right]$, and $T$ is bounded as a map from $\mathcal{H}_{1}$ into $\mathcal{H}_{2}$.

(2) If $B_{1}$ is reflexive, then range $(T) \in B\left[\Re_{2}\right]$.

(3) If $\mathfrak{B}_{1}$ is a Hilbert space, then $T\left[A \cap \mathcal{N}_{T}^{\perp}\right] \in B\left[\mathfrak{B}_{2}\right]$ for all $A \in B\left[\mathfrak{B}_{1}\right]$.

Proof. (1) Denote the norm on $\mathfrak{B}_{i}$ by $\|\cdot\|_{i}$. Let $\mathfrak{B}_{3}$ be the separable Banach space consisting of range(T) and the $\mathscr{B}_{2}$ norm. Define $T_{1}: \mathscr{B}_{1} \rightarrow \mathscr{B}_{3}$ by $T_{1} x=$ $T x_{\text {. Let }}\left\{x_{n}\right\}, n=1,2, \cdots$, be a dense subset of $\mathfrak{B}_{1}$. Since range $\left(T_{1}\right)$ is dense in $B_{3},\left\{T_{1} x_{n}\right\}, n=1,2, \cdots$, is dense in $B_{3}$.

For each $x_{n}$, define an element $F_{n}$ in $\mathscr{B}_{3}^{*}$ as follows. If $\left\|T x_{n}\right\|_{2}=0, F_{n}$ is the null element. If $\left\|T x_{n}\right\|_{2} \neq 0$, pick $F_{n}$ so that $\left\|F_{n}\right\|=1$ and $F_{n}\left(T x_{n}\right)=\left\|T x_{n}\right\|_{2}$. 
Let $\left\{a_{n}\right\}$ be a set of real scalars such that $a_{n}=0$ if $\left\|T x_{n}\right\|_{2}=0$, while otherwise $a_{n}>0$, and $\Sigma_{n} a_{n}=1$. Define an inner product $\langle\cdot, \cdot\rangle_{3}$ and norm $\|\cdot\|_{3}$ on $\mathfrak{B}_{3}$ by $\langle u, v\rangle_{3}=\Sigma_{n} a_{n} F_{n}(u) F_{n}(v)$. The norm obtained from this inner product is obviously weaker than the $\|\cdot\|_{2}$ norm, since $\|T u\|_{3}^{2} \leq \sup _{n} F_{n}^{2}(T u)=\|T u\|_{2}^{2}$. Let $\mathcal{H}_{3}$ be the Hilbert space obtained by completing $\mathfrak{B}_{3}$ under the norm $\|\cdot\|_{3}$. Let $I_{1}: \mathfrak{B}_{3} \rightarrow \mathcal{H}_{3}$ be the natural injection map. Since the $\mathfrak{B}_{3}$ norm is stronger than the $\mathcal{H}_{3}$ norm, $I_{1}$ is bounded, linear, and one-to-one; by Kuratowski's theorem, $I_{1}[A] \in B\left[\mathcal{H}_{3}\right]$ whenever $A \in B\left[\mathscr{B}_{3}\right]$. Hence $A \in B\left[\mathcal{H}_{3}\right]$, so that $B\left[\mathscr{B}_{3}\right] \subset B\left[\mathcal{H}_{3}\right]$. If $C \in B\left[\mathcal{H}_{3}\right]$, then $\mathfrak{B}_{3} \cap C=I_{1}^{-1}\left[\mathfrak{B}_{3} \cap C\right]$; since $\mathfrak{B}_{3} \cap C$ belongs to $B\left[\mathcal{H}_{3}\right], I_{1}^{-1}\left[\mathfrak{B}_{3} \cap C\right] \in B\left[\mathfrak{B}_{3}\right]$. Thus, $B\left[\mathscr{B}_{3}\right]=\mathscr{B}_{3} \cap B\left[\mathcal{H}_{3}\right]$.

Consider $T_{1}^{*} F_{n^{*}}\left\|T_{1}^{*} F_{n}\right\| \leq\|T\|$ since $\left\|F_{n}\right\|=1$. Moreover, $\|T x\|_{2}=$ $\sup _{n}\left|\left[T_{1}^{*} F_{n}\right](x)\right| \leq \sup _{n}\left\|T_{1}^{*} F_{n}\right\|\|x\|_{1}$. Hence $\|T\|=\sup _{n}\left\|T_{1}^{*} F_{n}\right\|$. Let $\left\{L_{n}\right\}$, $n=1,2, \cdots$, be a set of elements in $B_{1}^{*}$ selected as follows. For $n$ such that $a_{n}=0$, pick $L_{n}$ such that $\left\|L_{n}\right\|=1$ and $L_{n}\left(x_{n}\right)=\left\|x_{n}\right\|_{1}$. For $n$ such that $a_{n} \neq 0$, define $L_{n}=\left\|T_{1}^{*} F_{n}\right\|^{-1} T_{1}^{*} F_{n}$. Define scalars $\left\{\beta_{n}\right\}, n=1,2, \cdots$, such that $\beta_{n}>0$, all $n, 2 \beta_{n}=\alpha_{n}$ if $\alpha_{n} \neq 0$, and $\Sigma_{n=1}^{\infty} \beta_{n} \leq 1$. Define an inner product $\langle\cdot, \cdot \cdot\rangle_{1}$ on $\beta_{1}$ by $\langle u, v\rangle_{1}^{n}=\Sigma_{n=1}^{\infty} \beta_{n} L_{n}(u) L_{n}(v)$. Note that $\langle u, u\rangle_{1} \leq \Sigma_{n=1}^{\infty} \beta_{n}\|u\|_{1}^{2} \leq$ $\|u\|_{1}^{2}$. Let $\mathcal{H}_{1}$ be the separable Hilbert space obtained by completing $\mathfrak{B}_{1}$ under the norm obtained from the inner product $\langle\cdot, \cdot\rangle_{1}$. Using the natural injection of $\mathfrak{B}_{1}$ into $\mathcal{H}_{1}$ and Kuratowski's theorem, one obtains $B\left[\mathscr{B}_{1}\right] \subset B\left[\mathcal{H}_{1}\right], B\left[\mathscr{B}_{1}\right]=$ $\mathfrak{B}_{1} \cap B\left[\mathcal{H}_{1}\right]$.

Consider $T_{1}$ as a map from $\mathcal{H}_{1}$ into $\mathcal{H}_{3}$. For $u$ in $\mathfrak{B}_{1}$, one has

$$
\begin{aligned}
2\|T\|^{2}\langle u, u\rangle_{1} & \geq 2\|T\|^{2}\left\{1 / 2 \sum_{n} a_{n}\left\|T_{1}^{*} F_{n}\right\|^{-2}\left[T_{1}^{*} F_{n}\right]^{2}(u)\right\} \\
& \geq \sum_{n} a_{n} F_{n}^{2}\left(T_{1} u\right)=\left\|T_{1} u\right\|_{3}^{2},
\end{aligned}
$$

where the summations are over all $n$ such that $\alpha_{n} \neq 0$. Thus, $T_{1}$ is a bounded linear map from $B_{1}$ into $\mathcal{H}_{3}$, and can be extended by continuity to a bounded linear operator on $\mathcal{H}_{1}$.

(2) Since $\mathfrak{B}_{1}$ is reflexive, the convex set $A_{n}=\left\{x \in \mathfrak{B}_{1}:\|x\|_{1} \leq n\right\}$ is weakly compact, and hence $T\left[A_{n}\right]$ is closed in the norm topology. Thus $\bar{T}\left[B_{1}\right]=$ $\cup_{n} T\left[A_{n}\right]$ is in $B\left[\mathscr{B}_{2}\right]$.

(3) The operator $T$ is one-to-one on the Hilbert space consisting of $r_{T}^{\perp}$ and the $\mathfrak{B}_{1}$ inner product. For $A \in B\left[\mathfrak{B}_{1}\right], A \cap r_{T}^{1}$ is a Borel set in $r_{T^{*}}^{1}$ The fact that $T\left[A \cap \gamma_{T}\right]$ is in $B\left[\Re_{2}\right]$ follows from Lemma 2 .

5. Necessary and sufficient conditions for $\mu(G)=1$. There are many problems in which one will wish to determine if $\mu(G)=1$ for some measurable subgroup $G$. 
Theorem 2 gives necessary and sufficient conditions for $\mu(G)=1$ for a large class of subgroups.

Theorem 2. Suppose $T: \mathcal{H} \rightarrow \mathfrak{B}$ is a bounded linear operator. If $\mu$ is a Gaussian measure on $B[\Re]$, with mean element $m$, then

(a) $\mu[$ range $(T)]=0$ or 1 .

(b) $\mu[$ range $(T)]=1$ if and only if there exists a Gaussian measure $\nu$ on $B[\mathcal{H}]$ such that $\mu[A]=\nu\{x: T x \in A\}$ for all $A$ in $B[B]$.

(c) Let $\mathcal{H}_{1}$ be a separable Hilbert space containing $B$ as a dense linear manifold and such that $\mathfrak{B} \in B[\mathcal{H}], B[\mathfrak{B}]=\mathscr{B} \cap B[\mathcal{H}]$. Let $\mu_{1}$ be the Gaussian measure on $B\left[\mathcal{H}_{1}\right]$ defined by $\mu_{1}[A]=\mu[\Re \cap A], A \in B\left[\mathcal{H}_{1}\right]$ : Then $\mu[\operatorname{range}(T)]=1$ if and only if $m \in \operatorname{range}(T)$ and there exists a covariance operator $S$ in $\mathcal{H}_{1}$ such that $K_{1}=T S T_{1}^{*}$, where $K_{1}$ is the covariance operator of $\mu_{1}$ and $T_{1}^{*}$ is the Hilbert space adjoint of $T, T_{1}^{*}: \mathcal{H}_{1} \rightarrow \mathcal{H}$.

Proof. Part (a) follows from Theorem 1 and Lemma 3. To prove (b), we first note that if such a measure $\nu$ exists, then obviously $\mu[$ range $(T)]=1$. Thus, sup-

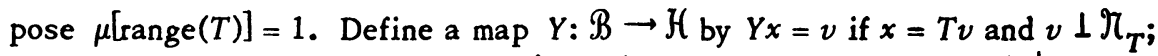
$Y x=0$ if $x \notin \operatorname{range}(T)$. For $A \in B[\mathcal{H}], Y^{-1}(A)=\left\{x: x=T v, v \in A \cap \gamma_{T}^{\frac{1}{2}}\right\}$ if $0 \notin A$. If $0 \in A$, then $Y^{-1}(A)=T\left[A \cap \Re_{T}^{\perp}\right] \cup\{x: x \notin$ range $(T)\}$. In both cases, $Y^{-1}(A)$ belongs to $B[\Re]$, by Lemma 3 , and hence $Y$ is $B[\Re] / B[\mathcal{H}]$ measurable. $Y$ thus induces a measure $\nu$ on $B[\mathcal{H}]$ from $\mu ; \nu(A)=\mu\{x: Y x \in A\} . \nu(\mathcal{H})=$ $\mu\{x: Y x \in \mathcal{H}\}=1$; also, $\nu\left[\mathcal{\eta}_{T}^{1}\right]=\mu\left\{x: Y x \perp \mathcal{K}_{T}\right\}=\mu[$ range $(T)]=1$. To see that $\nu$ is Gaussian, let $u$ be any element of $\overline{\operatorname{range}\left(T^{*}\right)}$; then there exists $\left\{u_{n}\right\} \subset \mathscr{B}^{*}$ such that $T^{*} u_{n} \rightarrow u$. Thus $\nu\{y:\langle y, u\rangle<k\}=\mu\{x:\langle Y x, u\rangle<k\}=\mu\left\{x: \lim _{n}\left\langle Y x, T^{*} u_{n}\right\rangle<\right.$ $k\}=\mu\left\{x: \lim _{n} u_{n}(T Y x)<k\right\}=\mu\left\{x: \lim _{n} u_{n}(x)<k\right\}$, since range $(T)=\{x: T Y x=x\}$. As the a.e. limit of a sequence of Gaussian random variables, the random variable $f_{u}: x \rightarrow\langle x, u\rangle$ is Gaussian with respect to $\nu . \nu$ is thus a Gaussian probability measure. To see that $\mu$ is induced from $\nu$ by $T$, one notes that $\mu\{x: x \in A\}=$ $\mu\{x: x \in A \cap \operatorname{range}(T)\}=\mu\{x: T Y x \in A\}=\nu\{x: T x \in A\}$.

To prove (c), we note that $\mu[$ range $(T)]=1 \Leftrightarrow \mu_{1}[\operatorname{range}(T)]=1$. Thus, if $\mu[$ range $(T)]=1$, let $\nu$ be the Gaussian measure on $B[\mathcal{H}\}$ such that $\mu_{1}=\nu \circ T^{-1}$. Let $S$ and $y$ be the covariance operator and mean element of $\nu$. We first note that $T y=m$, since $\int_{\mathcal{H}_{1}}\langle x, u\rangle_{1} d \mu_{1}(x)=\int_{\mathcal{H}}\left\langle x, T_{1}^{*} u\right\rangle d \nu(x)$, all $u$ in $\mathcal{H}_{1}$, where $\langle\cdot, \cdot\rangle_{1}$ is

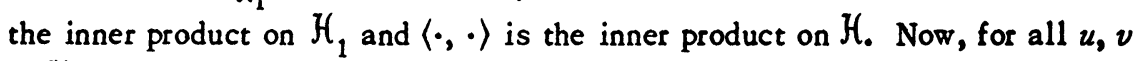
in $\mathcal{H}_{1}$,

$$
\begin{aligned}
\left\langle K_{1} u, v\right\rangle_{1} & =\int_{\mathcal{H}_{1}}\langle x-m, u\rangle_{1}\langle x-m, v\rangle_{1} d \mu_{1}(x) \\
& =\int_{\mathcal{K}}\left\langle x-y, T_{1}^{*} u\right\rangle\left\langle x-y, T_{1}^{*} v\right\rangle d \nu(x)=\left\langle T S T_{1}^{*} u, v\right\rangle_{1} .
\end{aligned}
$$


Hence, $K_{1}=T S T_{1}^{*}$. Conversely, if $K_{1}=T S T_{1}^{*}, S$ a covariance operator in $\mathcal{H}$, and $m=T y$ for $y \in \mathcal{H}$, we define $\nu$ to be the Gaussian measure on $B[\mathcal{H}]$ with covariance operator $S$ and mean element $y$. The map $T: \mathcal{H} \rightarrow \mathcal{H}_{1}$ induces from $\nu$ a Gaussian measure on $B\left[\mathcal{H}_{1}\right]$ having covariance operator $T S T_{1}^{*}$ and mean element $m$; since a Gaussian measure on $\mathcal{H}_{1}$ is uniquely specified by its covariance operator and mean element, this measure must be $\mu_{1}$.

Some applications of Theorem 2 are given in the next section. However, it may be of interest to note here the following. Suppose $\mu$ is a Gaussian measure on $B[\mathcal{H}]$, with covariance operator $K$. Part (c) of Theorem 2 shows that $\mu\left\{x: x+m \in\right.$ range $\left.\left(K^{1 / 2}\right)\right\}=0$ for all $m \in \mathcal{H}$. This property is not shared, for arbitrary Gaussian $\mu$, by all subgroups $G \in B[\mathcal{H}]$ such that $\mu(G)=0$. For example, if $\mu$ is a zero-mean Gaussian measure such that $\mu[$ range $(T)]=1$ for $T$ a bounded and linear map in $\mathcal{H}$, then $\mu_{1}$ [range $\left.(T)\right]=0, \mu_{1}\{x: x-m \in \operatorname{range}(T)\}=1$, where $\mu_{1}$ is the translate of $\mu$ by $m, m \notin \operatorname{range}(T)$. However, it is clear from Theorem 2 that if $\mu$ is a zero-mean Gaussian measure and $T: \mathcal{H} \rightarrow \mathcal{H}$ is bounded and linear, then $\mu[\operatorname{range}(T)]=0 \Leftrightarrow \mu\{x: x+m \in \operatorname{range}(T)\}=0$ for all $m \in \mathcal{H}$. It would be interesting to know whether this holds for all subgroups in $B[\mathcal{H}]$. If so, the method used to prove Theorem 1 shows that a similar result would hold for any subgroup in $B[\Re]$.

Part (c) of Theorem 2 is an extension of a result by Pitcher [18], who proved the following. Suppose $\mu$ is a zero-mean Gaussian measure on $B[\mathcal{H}]$, with covariance operator $K$. Suppose $K=T S T$, where $T$ and $S$ are linear, bounded, nonnegative, selfadjoint, and Hilbert-Schmidt operators in $\mathcal{H}$, with $T$ strictly positive. Then $\mu[\mathrm{range}(T)]=0$ or 1 , and $\mu[\mathrm{range}(T)]=1$ if and only if $S$ is traceclass. His method of proof requires the assumptions that $K=T S T$, that $\overline{\operatorname{range}(T)}=$ $\overline{\text { range }(S)}=\mathcal{H}$, and that $S$ is selfadjoint and compact.

The following theorem generalizes (a) and (b) of Theorem 2 to two Banach spaces.

Theorem 3. Suppose $\mathfrak{B}_{i}(i=1,2)$ is a real separable Banach space, and that $T: \mathscr{B}_{1} \rightarrow \mathscr{B}_{2}$ is a bounded linear operator. Suppose that either $\mathfrak{B}_{1}$ is reflexive, or else $T$ is one-to-one. Let $\mu$ be a Gaussian measure on $B\left[\mathscr{B}_{2}\right]$. Then $\operatorname{range}(T) \in B\left[\Re_{2}\right]$, and $\mu[$ range $(T)]=0$ or 1 . Moreover, $\mu[$ range $(T)]=1$ if and only if there exists a Gaussian measure $\nu$ on $B\left[\mathscr{B}_{1}\right]$ sucb that $\mu$ is induced from $\nu$ by $T$.

Proof. First consider the case where $T$ is one-to-one. It is clear that we need only prove the existence of $\nu$ as defined in the theorem when $\mu[$ range $(T)]=1$. Since $T^{-1}$ exists, it is obvious that there exists a probability measure $\nu$ on $B\left[\mathscr{B}_{1}\right]$ such that $\nu(A)=\mu\left\{x: T^{-1} x \in A\right\}$, and that $T$ induces $\mu$ from $\nu$. We show $\nu$ is Gaussian. 
Let $y$ be any element of $\mathfrak{B}_{1}^{*}$ belonging to range $\left(T^{*}\right)$; say $y=T^{*} v$. Then, $\nu\{x: y(x)<k\}=\mu\left\{x: y\left(T^{-1} x\right)<k\right\}=\mu\left\{x: T^{*} v\left(T^{-1} x\right)<k\right\}=\mu\{x: v(x)<k\}$. This number is uniquely defined, since if $T^{*} v=T^{*} z$, then $T^{*} v\left(T^{-1} x\right)=T^{*} z\left(T^{-1} x\right)$ for all $x$ in range $(T)$, so that $v(x)=z(x)$ with $\mu$-measure one. Hence, one sees that $y(x)$ is Gaussian with respect to $\nu$ for all $y$ in range $\left(T^{*}\right)$. Range $\left(T^{*}\right)$ is weak ${ }^{*}$-dense in $\mathfrak{B}_{1}^{*}$, since $T$ is one-to-one. Thus, if $y \in \mathfrak{B}_{1}^{*}$ is not in range $\left(T^{*}\right)$, then there exists a sequence $\left\{y_{n}\right\} \subset$ range $\left(T^{*}\right)$ such that $y_{n}(x) \rightarrow y(x)$ for all $x \in \mathfrak{B}_{1}$. Hence $y$ is the a.e. $(\nu)$ limit of a sequence of Gaussian random variables, and thus is Gaussian.

For the case where $B_{1}$ is reflexive, with $T$ not necessarily one-to-one, suppose that $\mu[$ range $(T)]=1$. From Lemma 3 , one can imbed $\mathfrak{B}_{1}$ densely in a separable Hilbert space $\mathcal{H}_{1}$ such that $B\left[\mathscr{B}_{1}\right]=\mathscr{B}_{1} \cap B\left[\mathcal{H}_{1}\right]$, while $\overline{\text { range }(T)}$ is imbedded densely in a separable Hilbert space $\mathcal{H}_{2}$ with $B[\overline{\operatorname{range}(T)}]=\overline{\operatorname{range}(T)} \cap B\left[\mathcal{H}_{2}\right]$, and $T$ can be extended by continuity to a bounded linear operator $T_{1}: \mathcal{H}_{1} \rightarrow \mathcal{H}_{2}$. Define $Y: \mathcal{H}_{2} \rightarrow \mathcal{H}_{1}$ by $Y x=v$ if $x=T_{1} v$ and $v \perp \pi_{T_{1}}$, while $Y x=0$ if $x \notin$ range $\left(T_{1}\right)$. Define $\mu^{\prime}$ on $B\left[\mathcal{H}_{2}\right]$ by $\mu^{\prime}(A)=\mu\left(\Re_{2} \stackrel{1}{\cap} A\right)$. The procedure used to prove (b) of Theorem 2 shows that there exists a Gaussian measure $\nu$ on $B\left[\mathcal{H}_{1}\right]$ such that $\mu^{\prime}(A)=\nu\left\{x: T_{1} x \in A\right\}$ for $A$ in $B\left[\mathcal{H}_{2}\right] . \nu\left(\mathscr{B}_{1}\right)=\nu\left\{x: T_{1} x \in \mathscr{B}_{2}\right\}=$ $\mu^{\prime}\left(\mathscr{B}_{2}\right)=1$. Let $\nu_{0}$ be the restriction of $\nu$ to $B\left[\mathscr{B}_{1}\right]$. If $u \in \Re_{1}^{*}$ belongs to $\mathcal{H}_{1}^{*}$, then $u$ is a Gaussian random variable with respect to $\nu_{0}$, since $\nu$ is Gaussian. Hence $\nu_{0}$ is Gaussian provided $\mathcal{H}_{1}^{*}$ is dense in $\mathfrak{B}_{1}^{*}$. Suppose there exists $f$ in $\mathfrak{B}_{1}^{* *}$ such that $f(v)=0$ for all $v$ in $\mathcal{H}_{1}^{*}$. Since $\mathfrak{B}_{1}$ is dense in $\mathcal{H}_{1}$ and $\mathfrak{B}_{1}$ is isometrically isomorphic to $\mathfrak{B}_{1}^{* *}, \mathfrak{B}_{1}^{* *}$ is dense in $\mathfrak{H}_{1}^{* *}$ and hence $f$ must be the null element of $\mathfrak{B}_{1}^{* *}$. Thus $\mathcal{H}_{1}^{*}$ is dense in $\mathfrak{B}_{1}^{*}$, so that $\nu_{0}$ is Gaussian, with $\mu(A)=$ $\nu_{0}\{x: T x \in A\}$ for $A \in B\left[\Re_{2}\right]$. The remainder of the proof is obvious.

Theorem 3 extends the following well-known result: If $\mu$ is a Gaussian measure on $B\left[\mathscr{B}_{1}\right]$, and $T: \mathscr{B}_{1} \rightarrow \mathscr{B}_{2}$ is bounded and linear, then the measure on $B\left[\Re_{2}\right]$ induced from $\mu$ by $T$ is also Gaussian. Thus if $T^{-1}$ exists, then Theorem 3 shows that for a Gaussian measure $\mu$ on $B\left[\mathfrak{B}_{2}\right], \mu\left[\operatorname{domain}\left(T^{-1}\right)\right]=0$ or 1 ; if $\mu\left[\right.$ domain $\left.\left(T^{-1}\right)\right]=1$, then the measure induced from $\mu$ by $T^{-1}$ is Gaussian. If $T^{-1}$ does not exist, but $B_{1}$ is reflexive, we still have that $\mu[\operatorname{range}(T)]=1$ if and only if there exists a Gaussian measure on $B\left[\mathfrak{B}_{1}\right]$ from which $\mu$ is induced by $T$.

6. Applications to Gaussian measures and Gaussian stochastic processes. In this section, the preceding theorems are applied to obtain a number of zeroone statements for Gaussian measures and Gaussian stochastic processes. Included are results on convergence of sequences, convergence of series, and analytical properties of sample paths.

All processes and random variables considered are real-valued, and defined on a probability space $(\Omega, \beta, P)$. T will denote a compact interval. $L_{p}$ or $L_{p}[T]$ will denote the space of equivalence classes of real-valued Lebesgue- 
measurable functions on $T$ such that $\int_{T}\left|f_{t}\right|^{p} d t<\infty$. $l_{p}$ denotes the set of all $p$ summable real-valued sequences; $C$ or $C[T]$ will denote the space of real-valued functions defined and continuous on $T$. In applications involving a norm or a norm topology on these spaces, it is always assumed that the standard norm is used; e.g., $\|f\|=\sup _{t \in T}\left|f_{t}\right|$ for $C$.

Theorem 4. Suppose $\left\{Z_{n}\right\}, n=1,2, \cdots$, is a family of jointly Gaussian real random variables on a probability space $(\Omega, \beta, P)$. The following sets belong to $\beta$ and have P-measure 1 or 0 :

(1) $A=\left\{\omega: Z_{n}(\omega)\right.$ converges to some real number $\left.k(\omega)\right\}$,

(2) $A_{k}=\left\{\omega: Z_{n}(\omega)\right.$ converges to $\left.k\right\}$ for any fixed real number $k$,

(3) $B=\left\{\omega: \sup _{n}\left|Z_{n}(\omega)\right|<\infty, Z_{n}(\omega)\right.$ is not convergent $\}$,

(4) $C=\left\{\omega: \lim \inf \left|Z_{n}(\omega)\right|<\infty\right\}$,

(5) $D=\left\{\omega: \sup _{n}\left|Z_{n}(\omega)\right|=\infty,\left|Z_{n}(\omega)\right|\right.$ is not convergent $\}$,

(6) $F=\left\{\omega:\left|Z_{n}(\omega)\right|\right.$ converges to $\left.\infty\right\}$. Moreover, if $\left\{Z_{n}, n \geq 1\right\}$ is an independent family with $\left\{E Z_{n}, n \geq 1\right\}$ bounded, then $P(F)=1$ if and only if $\Sigma_{k \geq 1} \sigma_{k}^{-1}<\infty$, where $\sigma_{k}^{2}$ is the variance of $Z_{k}$.

Proof. Let $\left\{g_{n}\right\}, n=1,2, \cdots$, be a set of real numbers such that $\Sigma_{n} g_{n}^{2} E Z_{n}^{2}<\infty, \Sigma_{n} g_{n}^{2}<\infty$, and $g_{n}>0$ for $n \geq 1$. Define $Y(\omega)$ by $Y_{i}(\omega)=g_{i} Z_{i}(\omega)$. Let $\mathbf{c}$ denote the space of all convergent sequences of real numbers $x=$ $\left(x_{1}, x_{2}, \cdots\right)$. c is a separable Banach space under the norm $\|x\|=\sup _{n}\left|x_{n}\right|$. Suppose $x \in \mathrm{c}$. Then $\Sigma_{i}\left(g_{i} x_{i}\right)^{2} \leq\left(\Sigma_{i} g_{i}^{2}\right)\|x\|^{2}$. Thus we can define a bounded, linear, and one-to-one operator $G: \mathrm{c} \rightarrow l_{2}$ by $(G x)_{i}=g_{i} x_{i}$. We see that $\{\omega: Z(\omega) \in \mathrm{c}\}=\{\omega: Y(\omega) \in$ range $(G)\}$. Now we note that $Y$ induces from $P$ a Gaussian measure $\mu$ on $B\left[l_{2}\right], A=Y^{-1}$ [range $\left.(G)\right]$, and $P(A)=\mu$ (range $(G)$ ), proving the assertion for $A$.

For the set $A_{k}$, we consider the space $\mathrm{c}_{0}$ of real sequences that are convergent to zero. We note that $\omega \in A_{k}$ if and only if $Z_{n}(\omega)-k \rightarrow 0 . \mathbf{c}_{0}$ is a separable Banach space under the norm $\|x\|=\sup _{n}\left|x_{n}\right|$. Defining the operator $G: c_{0} \rightarrow l_{2}$ by $(G x)_{i}=g_{i} x_{i}$, one sees that $G$ is bounded, linear, and one-to-one. Let $Y(\omega)$ be defined by $Y_{i}(\omega)=g_{i}\left(Z_{i}(\omega)-k\right)$. $Y(\omega)$ is in $l_{2}$ a.e. $d P(\omega)$, thus induces a Gaussian measure on $B\left[l_{2}\right]$. Moreover, $Z_{n}(\omega)-k \rightarrow 0$ if and only if $Y(\omega) \in$ range $(G)$. The remainder is clear.

To prove the $0-1$ statement on $B$, we apply a $0-1$ law for Gaussian random variables due to Landau and Shepp [15], which states that $P\left[\sup _{n}\left|Z_{n}\right|<\infty\right]=1$ or 0 . Since $\left\{\omega: \sup _{n}\left|Z_{n}(\omega)\right|<\infty\right\}$ is the union of the disjoint sets $A$ and $B, P(B)=$ 0 or 1 . Next, let $\left\{Y_{n}, n \geq 1\right\}$ and $Y: \Omega \rightarrow l_{2}$ be defined as in the proof for the set $A$. Then $C=Y^{-1}\left(C^{\prime}\right)$, where

$$
C^{\prime}=\left\{x \in l_{2}: \lim \inf \left|x_{n} / g_{n}\right|<\infty\right\}=\bigcup_{m \geq 1} \bigcap_{N \geq 1} \bigcup_{k}\left\{x:\left\|G_{k} x\right\|<m\right\},
$$


with $G_{k}: l_{2} \rightarrow l_{2}$ the bounded linear operator defined by $\left(G_{k} x\right)_{i}=\delta_{i k} x_{k} g_{k}$. Hence, $C^{\prime} \in B\left[l_{2}\right]$ and is a linear manifold, so that $P(C)=\mu\left(C^{\prime}\right)=0$ or $1, \mu$ the Gaussian measure on $B\left[l_{2}\right]$ induced from $P$ by $Y$. The $0-1$ statement on $D$ now follows from the fact that $C$ is the union of the disjoint sets $A, B$, and $D$. Similarly, the 0-1 law for $F$ is obtained by noting that $\Omega$ is the union of the disjoint sets $C$ and $F$.

Suppose now that $\left\{Z_{n}, n \geq 1\right\}$ is an independent family, with $\left|E Z_{n}\right| \leq M<$ $\infty, n \geq 1$. One has $P(F)=1-\lim _{m \rightarrow \infty} P\left[\lim \sup _{k} A_{m, k}\right]$, where $A_{m, k}=$ $\left\{\omega:\left|Z_{k}(\omega)\right| \leq m\right\}$. Thus $P(F)=1$ if $P\left[\lim \sup _{k} A_{m, k}\right]=0$ for all $m \geq 1$. Conversely, if $P(F)=1$, then $P\left[\lim \sup _{k} A_{m, k}\right]=0$ for all $m \geq 1$, since the sequence $\left\{P\left[\lim \sup _{k} A_{m, k}\right], m \geq 1\right\}$ is nondecreasing. Using the Borel-Cantelli lemmas and the independence of $\left\{Z_{n}, n \geq 1\right\}, P\left[\lim \sup _{k} A_{m, k}\right]=0$ if and only if $\Sigma_{k \geq 1} P\left(A_{m, k}\right)<\infty$. Hence, $P(F)=1$ if and only if $\Sigma_{k \geq 1} P\left(A_{m, k}\right)<\infty$ for all $m$. For each $m$ and $k$, one has $(2 / \pi)^{1 / 2} \exp \left[-(m+M)^{2} /\left(2 \sigma^{2}\right)\right] m / \sigma_{k} \leq P\left(A_{m, k}\right) \leq$ $(2 / \pi)^{1 / 2} m / \sigma_{k}$, where $\sigma^{2}=\inf _{k} \sigma_{k}^{2}$. Hence, $P(F)=1$ if $\Sigma_{k \geq 1} \sigma_{k}^{-1}<\infty$. Suppose $P(F)=1$; then by Fatou's lemma $\sigma_{k}^{2} \rightarrow \infty$, so that $\sigma^{2}>0$, and the lower bound on $P\left(A_{m, k}\right)$ shows that $\Sigma_{k \geq 1} \sigma_{k}^{-1}<\infty$. This completes the proof of the theorem.

We note that the proof of Theorem 4 shows that the set $F$ of the theorem has probability one if $\Sigma_{k \geq 1} \sigma_{k}^{-1}<\infty$, without the hypothesis of independence of $\left\{Z_{n}, n \geq 1\right\}$, and without requiring $\left\{E Z_{n}, n \geq 1\right\}$ to be bounded.

Corollary. Suppose $\left(X_{t}\right), t \in T$ (an interval), is a separable Gaussian process, with $t_{0} \in T$. Then $\lim _{t \mid t_{0}} X_{t}$ exists with probability one or zero; if the first alternative bolds, then $\lim _{t t_{0}} X_{t}=k$ with probability one or zero for any

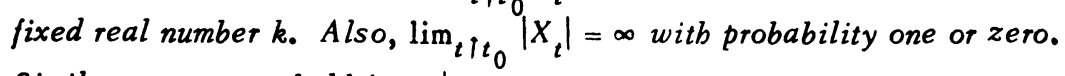
Similar statements bold for $t \downarrow t_{0}{ }^{\circ}$

Proof. There exists a monotone sequence $\left\{t_{n}, n \geq 1\right\} \subset T$ such that $t_{n} \uparrow t_{0}$ and $\lim \inf _{t i t_{0}} X_{t}=\lim \inf _{n \rightarrow \infty} X_{t_{n}}, \lim \sup _{t \uparrow t_{0}} X_{t}=\lim \sup _{n \rightarrow \infty} X_{t_{n}}$ with probability one $\left[4\right.$, p. 55]. From Theorem $4, \lim _{n \rightarrow \infty} X_{t}$ exists with probability one or zero. The remainder of the proof is clear.

The corollary yields many $0-1$ statements on local path properties of a separable Gaussian process, including differentiability, continuity, and each of the possible types of discontinuity (removable, jump of finite amplitude, jump of infinite amplitude, oscillatory with bounded oscillations, oscillatory with unbounded oscillations, and both limits existing, infinite, and of the same algebraic sign). These $0-1$ laws, which hold for each point in the index set, are for fixed discontinuities. If $\left(X_{t}\right)$ is a mean-square-continuous separable Gaussian process on $T$, then any fixed discontinuity must be oscillatory. Thus, for such 
a process, we obtain the result (first obtained by Itô and Nisio [9]) that every point of $T$ is either a point of a.s. continuity, or else a point of a.s. oscillatory discontinuity. If $\left(X_{t}\right)$ is also stationary, this yields Dobrushin's result [3] that either almost all paths of $\left(X_{t}\right)$ are continuous on $T$, or else almost all paths have an oscillatory discontinuity at every point of $T$.

The following theorem treats the convergence of series involving Gaussian random variables, including the Karhunen-Loeve expansion of mean-square-continuous Gaussian processes.

Theorem 5. Let $\left\{Z_{n}\right\}, n=1,2, \cdots$, be a family of jointly Gaussian random variables on a probability space $(\Omega, \beta, P)$. Let $\left\{e_{n}\right\}$ be a set of functions defined and continuous on the interval $T$. Define, for $1 \leq p<\infty$,

$A=\left\{\omega: \Sigma_{1}^{N} Z_{n}(\omega) e_{n}(t)\right.$ converges uniformly on $\left.T\right\}$,

$B_{p}=\left\{\omega: \Sigma_{1}^{N}\left[Z_{n}(\omega)\right]^{p} e_{n}(t)\right.$ converges absolutely and uniformly on $\left.T\right\}$,

$C_{p}=\left\{\omega: \Sigma_{1}^{\infty}\left|Z_{n}(\omega)\right|^{p}<\infty\right\}$.

Then $A, B_{p}$, and $C_{p}$ each belongs to $\beta$, each bas probability one or zero, and $P\left[C_{p}\right]=1$ if and only if $\Sigma_{1}^{\infty}\left[E\left(Z_{n}^{2}(\omega)\right)\right]^{p / 2}<\infty$.

Proof. Let $g_{n}=n^{-1}$ if $E Z_{n}^{2}(\omega) \leq 1$, and $g_{n}=n^{-1}\left[E Z_{n}^{2}(\omega)\right]^{-1}$ otherwise. Let $Y_{n}=g_{n} Z_{n}$; then $Y(\omega) \equiv\left(Y_{1}(\omega), Y_{2}(\omega), \cdots\right) \in l_{2}$ a.e. $d P(\omega)$. Let $\Omega_{1} \equiv$ $\left\{\omega: Y(\omega) \in l_{2}\right\}$. $Y: \Omega_{1} \rightarrow l_{2}$ induces a probability measure $\mu_{Y}$ on $B\left[l_{2}\right] ; \mu_{Y}$ is Gaussian, since if $\Sigma\left|x_{k}\right|^{2}<\infty, \Sigma x_{k} Y_{k}$ is a Gaussian random variable as the a.e. limit of a sequence of Gaussian random variables. If $\Sigma_{n}\left|x_{n}\right|^{p}<\infty, p \geq 1$, then $\Sigma_{n} n^{-2}\left|x_{n}\right|^{2} \leq \sup _{m}\left|x_{m}\right|^{2} \Sigma_{n} n^{-2} \leq 2\|x\|_{p}^{2}$. Hence we define the bounded, linear, and one-to-one operator $G_{p}: l_{p} \rightarrow l_{2}$ by $\left(G_{p} x\right)_{n}=g_{n} x_{n}$ for $x \in l_{p}$. Define

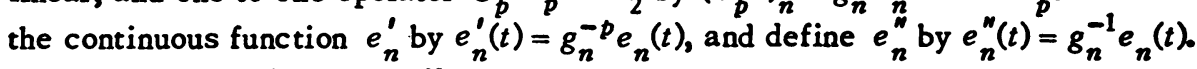
Note that $A=Y^{-1}\left\{x \in l_{2}: \Sigma_{1}^{N} x_{n} e_{n}^{\prime \prime}(t)\right.$ converges uniformly on $\left.T\right\}, B_{p}=$ $Y^{-1}\left\{x \in l_{2}: \Sigma_{1}^{N}\left|x_{n}\right|^{p} e_{n}^{\prime}(t)\right.$ converges absolutely and uniformly on $\left.T\right\}$, and $C_{p}=$ $Y^{-1}$ [range $\left(G_{p}\right)$ ]. To show that $A$ is in $\beta$ and $P(A)=0$ or 1 , we note that the set of $\omega$ for which the series converges uniformly is

$$
A=\bigcap_{N \geq 1} \bigcup_{M \geq 1} \bigcap_{m>n>M} \bigcap_{t \in S}\left\{\omega:\left|\sum_{k=n+1}^{m} e_{k}(t) Z_{k}(\omega)\right|<\frac{1}{N}\right\},
$$

where $S$ is a countable dense subset of $T$. But $A=Y^{-1}[D]$, where

$$
D=\bigcap_{N \geq 1} \bigcup_{M \geq 1} \bigcap_{m>n>M} \bigcap_{t \in S}\left\{x:\left|\sum_{k=n+1}^{m} e_{k}^{\prime \prime}(t) x_{k}\right|<\frac{1}{N}\right\} .
$$

$D$ is obviously in $B\left[l_{2}\right]$, since $\left\{x:\left|\Sigma_{k \in I} \gamma_{k} x_{k}\right|<1 / M\right\} \in B\left[l_{2}\right]$ for any finite index set $I$ and scalars $\left\{\gamma_{k}\right\}$. D is also a linear manifold. Hence $P[A]=\mu_{Y}[D]=0$ or 
$1, \mu_{Y}$ the measure induced from $P$ by $Y$. Similarly, using the inequality $|a+b|^{p} \leq$ $2^{p}\left(|a|^{p}+|b|^{p}\right), P\left(B_{p}\right)=0$ or 1 . It is clear that $C_{p}$ belongs to $\beta$ and that $P\left(C_{p}\right)=$ 0 or 1 . Finally, $P\left(C_{p}\right)=1$ if and only if the paths of $\left(Z_{n}\right)$ induce a Gaussian measure on $B\left[l_{p}\right]$; the results of [20], [21] show that this occurs if and only if $\Sigma_{n}\left[E Z_{n}^{2}(\omega)\right]^{p / 2}<\infty$.

The result for the set $A$ of the theorem shows that the Karhunen-Loeve expansion of a mean-square-continuous Gaussian process converges uniformly with probability one or zero. It is known ([6], [10]) that this occurs with probability one (for a separable process) if and only if almost all paths of $\left(X_{t}\right)$ are continuous on $T$. The following theorem gives results on analytic properties of sample paths when the paths are almost all continuous.

Theorem 6. Suppose $\mu$ is a Gaussian measure on the Borel $\sigma$-field of $C[T]$. The following sets belong to $B[C[T]]$ and bave $\mu$-measure zero or one: $p \in[1, \infty)$,

(1) $A C^{p}[T] \equiv\left\{x: x\right.$ is absolutely continuous with $L_{p}$ derivative $\}$, any fixed

(2) $C^{n}[T]=\{x: x$ is n-times continuously differentiable on $T\}$.

Proof. (1) Let $Q_{p}$ denote the real separable Banach space with elements $(a, x), \alpha$ a real scalar, $x$ in $L_{p}[T]$, and with norm defined by $\|(\alpha, x)\|=|\alpha|+\|x\|_{p}$. Let $S: Q_{p} \rightarrow C[T]$ be defined by $S(a, x)_{t}=a+\int_{a}^{t} x(s) d s$, where $T=[a, b] . S$ is a one-to-one bounded linear map, so that by Lemma 2 range $(S)$ is a Borel set in $C[T]$. Noting that range $(S)$ is a linear manifold and that range $(S)=A C^{p}[T]$, one sees that $\mu\left(A C^{p}[T]\right)=0$ or 1 .

(2) $C^{n}[T]$ is a real separable Banach space under the norm $\|x\|=$ $\Sigma_{i=0}^{n} \sup _{t \in T}\left|x_{t}^{(i)}\right|$. The natural injection of $C^{n}[T]$ into $C[T]$ is a bounded, linear, and one-to-one map. Thus $C^{n}[T]$ is a Borel-measurable linear manifold in $C[T]$, and the result follows.

If $p \geq 1$ and $\mu\left(A C^{p}\right)=1$, then Theorem 3 shows that the operation of differentiation induces from $\mu$ a Gaussian measure on $L_{p}$. For this, one notes that $s^{-1} f=\left(f(a), f^{(1)}\right)$ for $f \in A C^{p}, S$ as in the proof of Theorem 6. This induces a Gaussian measure on $B\left[Q_{p}\right]$, and projection into $L_{p}$ gives a Gaussian measure $\nu$ on $B\left[L_{p}\right], \nu(A)=\mu\left\{x \in A C^{p}: x^{(1)} \in A\right\}$ for $A \in B\left[L_{p}\right]$.

Before stating the next theorem, we establish a lemma essentially used by Pitcher [18].

Lemma 4. Suppose $\left(X_{t}\right), t \in T$, is a measurable Gaussian stochastic process. If almost all sample paths of $\left(X_{t}\right)$ belong to $L_{p}[T], 1 \leq p<\infty$, then $\left(X_{t}\right)$ induces a Gaussian measure $\mu$ on $B\left[L_{p}\right]$, defined by $\mu(A)=P\{\omega: X(\omega) \in A\}$, where $X(\omega)$ is the sample path of $\left(X_{t}\right)$ evaluated at $\omega$.

Proof. To see that $X: \Omega \rightarrow L_{p}$ is $\beta / B\left[L_{p}\right]$ measurable, one notes that by 
Fubini's theorem $\left\{\omega:\left[\int_{T}\left|X_{t}(\omega)\right|^{p} d t\right]^{1 / p}<\epsilon\right\}=X^{-1}\left\{x:\|x\|_{p}<\epsilon\right\}$ belongs to $\beta$. Since sets of the form $\left\{y:\|y\|_{p}<\epsilon\right\}, \epsilon>0$, form a neighborhood base at zero for the norm topology in $L_{p}, X^{-1}[A] \in \beta$ for $A \in B\left[L_{p}\right]$. Hence, $X$ induces a probability measure $\mu$ on $B\left[L_{p}\right]$. As noted by Pitcher [18], a result of Doob [4, Pp. 6465] shows that every bounded linear functional on $L_{p}$ is Gaussian with respect to $\mu$, so that $\mu$ is a Gaussian measure.

For the next result, we make the following definitions. $C_{\text {eq }}[T]$ is the set of all equivalence classes in $L_{1}[T]$ that contain an element of $C[T] ; C_{\text {eq }}^{n}[T]$ and $A C_{\text {eq }}^{p}[T]$ are defined similarly.

Theorem 7. Suppose $\left(X_{t}\right), t \in T$, is a measurable Gaussian stochastic process on a complete probability space $(\Omega, \beta, P)$. The following sets belong to $\dot{\beta}$ and bave probability zero or one:

(1) $\left\{\omega: X(\omega) \in L_{p}[T]\right\}$, any $p \geq 1$,

(2) $\left\{\omega: X(\omega) \in C_{\mathrm{eq}}^{p}[T]\right\}$,

(3) $\left\{\omega: X(\omega) \in C_{\text {eq }}^{n}[T]\right\}$, any $n \geq 1$,

(4) $\left\{\omega: X(\omega) \in A C_{\text {eq }}^{p}[T]\right\}$, any $p \geq 1$.

Proof. Define the function $g$ on $T$ by $g(t)=1$ if $E Z_{t}^{2}<1 ; g(t)=\left(E Z_{t}^{2}\right)^{-1}$ otherwise. Let $\left(Y_{t}\right)=\left(g_{t} X_{t}\right) ;\left(Y_{t}\right)$ is a measurable Gaussian process with almost all sample paths in $L_{1}$. Thus $Y$ induces from $P$ a Gaussian measure $\mu_{Y}$ on $B\left[L_{1}\right]$. Now let $G: L_{1} \rightarrow L_{1}$ be defined by $G v=g v, g$ as above. $G$ is bounded, linear, and one-to-one. Clearly, $\left\{\omega: X(\omega) \in L_{1}\right\}=\{\omega: Y(\omega) \in$ range $(G)\}$. Hence, $\left\{\omega: X(\omega) \in L_{1}\right\} \in \beta$, and $P\left\{\omega: X(\omega) \in L_{1}\right\}=\mu_{Y}\{$ range $(G)\}=0$ or 1 . For $p>1$, one uses the fact that $L_{1} \supset L_{p}$, and that the natural injection of $L_{p}$ into $L_{1}$ is a bounded, linear, and one-to-one operator.

To prove the theorem for the sets in (2)-(4), we first note that $C_{\mathrm{eq}}[T]$, $C_{e q}^{n}[T]$ and $A C_{e q}^{p}[T]$ are subsets of $L_{1}[T]$. From part (1), either almost all paths of $\left(X_{t}\right)$ belong to $L_{1}[T]$, or almost all do not belong to $L_{1}[T]$. If almost all paths lie outside $L_{1}$, then $X^{-1}[A] \in \beta$ for all subsets $A$ of $L_{1}$, since $(\Omega, \beta, P)$ is complete. Thus to prove the theorem, it is sufficient to show that the sets indicated in (2)-(4) of the theorem are elements of $\beta$, and that each set has probability one or zero, when almost all paths of $\left(X_{t}\right)$ belong to $L_{1}[T]$.

Thus, suppose that $P\left\{\omega: X(\omega) \in L_{1}\right\}=1$, and let $\mu_{X}$ be the Gaussian measure induced on $B\left[L_{1}\right]$ by $\left(X_{t}\right) ; \mu_{X}(A)=P\{\omega: X(\omega) \in A\}$. Let $W$ be the map taking $x$ in $C$ into its equivalence class in $L_{1}$. Since $\int_{T}\left|x_{t}\right| d t \leq T \sup _{t \in T}\left|x_{t}\right|, W$ is bounded and linear; $W$ is also one-to-one. Thus range $(W) \in B\left[L_{1}\right]$ by Lemma 2, and hence $\mu_{X}[$ range $(W)]=0$ or 1 . Since range $(W)=C_{e q}[T]$, this shows that $X^{-1}\left(C_{\text {eq }}\right) \in \beta$ and that $P\left[X^{-1}\left(C_{\text {eq }}\right)\right]=0$ or 1 .

To prove the remainder of the theorem, we note that in the proof of Theorem 
6 both $C^{n}[T]$ and $A C^{p}[T]$ were shown to be Borel linear manifolds in $C[T]$. Defining $W: C \rightarrow L_{1}$ as above, noting that $C_{\text {eq }}^{n}=W\left[B^{n}\right]$ and that $A C_{\text {eq }}^{p}=W\left[A C^{p}\right]$, the remainder of the proof is clear.

The statement on the set (1) of Theorem 7 does not require $(\Omega, \beta, P)$ to be complete.

The preceding theorems contain various zero-one laws for Gaussian processes. The problem of determining necessary and sufficient conditions for the alternatives is usually more difficult. Some general results in this direction are given in Theorem 2. The following theorem is an application of Theorem 1 and Theorem 2.

Theorem 8. Suppose that $\left(X_{t}\right), t \in(-\infty, \infty)$, is a measurable mean-squarecontinuous Gaussian process on a complete probability space $(\Omega, \beta, P)$. Suppose that $\left(X_{t}\right)$ is stationary with rational spectral density function $f$ and with zero mean. Let

$A \equiv$ ireal valued functions $x: x$ is absolutely continuous on $(-\infty, \infty)$, with derivative belonging to $L_{2}[T]$ for every compact interval $\left.T\right\}$, Then,

$A_{\text {eq }} \equiv\{$ real valued functions $x: x$ is equal a.e. (Lebesgue) to an element of $A\}$.

(1) $X^{-1}\left[A_{\text {eq }}\right] \in \beta$, and $P\left(X^{-1}\left[A_{\text {eq }}\right]\right)=0$ or 1 .

(2) If $\left(X_{t}\right)$ is separable, then $X^{-1}[A] \in \beta$, and $P\left(X^{-1}[A]\right)=0$ or 1 .

(3) $P\left(X^{-1}\left[A_{\text {eq }}\right]\right)=1$ if and only if $\int_{-\infty}^{\infty} \lambda^{2} f(\lambda) d \lambda<\infty$. If $\left(X_{t}\right)$ is separable, then this condition is also necessary and sufficient for $P\left(X^{-1}[A]\right)=1$.

Proof. A function is absolutely continuous on $(-\infty, \infty)$ if and only if it is absolutely continuous on every compact interval. The fact that $X^{-1}\left[A_{e q}\right] \in \beta$ and has probability zero or one thus follows from Theorem 7.

Let $T$ be any fixed compact interval. Let $R_{T}$ and $R_{0 T}$ be the integral operators in $L_{2}[T]$ having kernels $R(t, s)$ and $R_{0}(t, s)$, defined by

$$
\begin{aligned}
R(t, s) & =\int_{-\infty}^{\infty} f(\lambda) \exp (i \lambda(t-s)) d \lambda, \\
R_{0}(t, s) & =\int_{-\infty}^{\infty}\left(\lambda^{2}+1\right)^{-1} \exp (i \lambda(t-s)) d \lambda .
\end{aligned}
$$

Using a result of Hajek $[8, \S 7]$, one can show that $R_{T}=R_{0 T}^{1 / 2} W R_{0 T}^{1 / 2}$ for $W$ traceclass if and only if $\int_{-\infty}^{\infty}\left(\lambda^{2}+1\right) f(\lambda) d \lambda<\infty$. It is known [1] that the range of $\left(R_{0 T}^{1 / 2}\right)$ consists of all elements of $L_{2}[T]$ that are equal a.e. $d t$ to an absolutely continuous function with $L_{2}[T]$ derivative. From Theorem 2, one concludes that $P\left(X^{-1}\left[A_{\text {eq }}\right]\right)=1$ if and only if $\int_{-\infty}^{\infty} \lambda^{2} f(\lambda) d \lambda<\infty$.

To prove the statements regarding $A$, we first note that $X^{-1}[A] \subset X^{-1}\left[A_{\text {eq }}\right]$. Hence, if $\int_{-\infty}^{\infty} \lambda^{2} f(\lambda) d \lambda=\infty$, then $X^{-1}[A] \in \beta$, since $(\Omega, \beta, P)$ is complete, and 
$P\left(X^{-1}[A]\right)=0$. To complete the proof, it is sufficient to show that $\int_{-\infty}^{\infty} \lambda^{2} f(\lambda) d \lambda<\infty$ and $\left(X_{t}\right)$ separable imply that almost all paths of $\left(X_{t}\right)$ are absolutely continuous. This has been proved by Doob [4, pp. 535-537].

As noted in the proof of the theorem, this result is an extension in one direction of a result of Doob, which states that $\left(X_{t}\right)$ has absolutely continuous paths if $\int_{-\infty}^{\infty} \lambda^{2} d F(\lambda)<\infty, F$ the spectral distribution function of $\left(X_{t}\right)$, and $\left(X_{t}\right)$ separable [4, pp. 535-537]. However, the result given here is restricted to the case where $\left(X_{t}\right)$ has a spectral density, and this spectral density must be rational. The requirement that $\left(X_{t}\right)$ be Gaussian is not assumed by Doob; the process need only be separable, measurable, mean-square-continuous, and wide-sense stationary. Under these same assumptions, with the process also assumed to have a spectral density function, which is rational, the proof of Theorem 8 can be used to show that almost all sample, path derivatives belong to $L_{2}[T]$ for all compact intervals $T$ whenever $\int_{-\infty}^{\infty} \lambda^{2} f(\lambda) d \lambda<\infty$; i.e., normality is not required.

Theorem 8 is proved for zero-mean Gaussian processes. If the process has mean function $m$, then application of Theorem 2 shows that the necessary and sufficient condition for $A_{\text {eq }}$ (or $A$, if separability of $\left(X_{t}\right)$ is assumed) to have probability one is that $\int_{-\infty}^{\infty} \lambda^{2} f(\lambda) d \lambda<\infty$ and $m_{T} \in$ range $\left(R_{0 T}^{1 / 2}\right)$ for each compact interval $T$, where $m_{T}(t)=m(t), t \in T, m_{T}(t)=0, t \notin T$. A sufficient condition for $m_{T}$ to be in range $\left(R_{0 T}^{1 / 2}\right)$ is that $\int_{\infty}^{\infty}\left|\hat{m}_{T}(\lambda)\right|^{2} \lambda^{2} d \lambda<\infty[13]$, where $\hat{m}_{T}$ is the Fourier transform of $m_{T}$.

We discuss an application to information theory. Suppose that $\left(S_{t}\right),\left(N_{t}\right)$, $t \in T$ (compact interval), are measurable zero-mean Gaussian stochastic processes on a probability space $(\Omega, \beta, P)$. We assume that $\left(S_{t}\right)$ and $\left(N_{t}\right)$ are statistically independent, and that almost all sample paths belong to $L_{2}[T]$ for each process. Let $\mu_{S}, \mu_{N}$ and $\mu_{S+N}$ denote the Gaussian measures induced on $L_{2}[T]$ by $\left(S_{t}\right),\left(N_{t}\right)$ and $\left(S_{t}+N_{t}\right)$, respectively; e.g. $\mu_{S}(A)=P\{\omega: S(\omega) \in A\}, S(\omega)$ the sample path of $\left(S_{t}\right)$ evaluated at $\omega$. Let $\mu_{S, S+N}$ be the Gaussian measure induced on $B\left[L_{2} \times L_{2}\right]$ by $\left(S_{t}, S_{t}+N_{t}\right)$. The average mutual information (AMI) of $\left(S_{t}\right)$ and $\left(S_{t}+N_{t}\right)$ is defined as [7]

$$
\operatorname{AMI}(S, S+N)=\int_{L_{2} \times L_{2}} \log \left[\frac{d \mu_{S, S+N}}{d \mu_{S} d \mu_{S+N}}(x, y)\right] d \mu_{S, S+N}(x, y)
$$

if $\mu_{S, S+N} \sim \mu_{S} \otimes \mu_{S+N}$, and equal to $+\infty$ otherwise. This quantity is of much interest in many communication theory problems, where it represents the average information about the "signal" $\left(S_{t}\right)$, obtained by observing "signal plus noise" $\left(S_{t}+N_{t}\right)$. Hájek [8] has shown that for the case considered here, $\operatorname{AM}(S, S+N)<\infty$ $\Rightarrow K_{S}=K_{N}^{1 / 2} G K_{N}^{1 / 2}$ for a trace-class operator $G$, where $K_{S}$ and $K_{N}$ denote the covariance operators of $\left(S_{t}\right)$ and $\left(N_{t}\right)$. From this and Theorem 2 above, one sees that 
$\operatorname{AMI}(S, S+N)<\infty$ if and only if almost all sample paths of $\left(S_{t}\right)$ belong to the range of $K_{N^{*}}^{1 / 2}$ This result has been obtained by $\mathrm{Pitcher}[18]$, under the assumption that $\mu_{S+N} \sim \mu_{N^{*}}$

7. Zero-one laws for non-Gaussian measures. Our results so far involve only Gaussian measures. However, if $\mu$ is a Gaussian measure on $B[B]$, and $\mu^{\prime}$ is any probability measure on $B[B]$ such that $\mu^{\prime}$ is absolutely continuous with respect to $\mu$, then obviously $\mu^{\prime}(G)=0$ or 1 for any subgroup $G$ belonging to $B_{\mu}[\Re]$. Moreover, $B_{\mu},[\Re] \supset B_{\mu}[\Re]$; if also $\mu$ is absolutely continuous with respect to $\mu^{\prime}$, then $\left.B_{\mu},[\Re]\right]^{\mu}=B_{\mu}[\Re]$. In this section, we consider a class of non-Gaussian measures for which this holds. Our results are based on the following lemma. The notation $\mu_{1} \ll \mu_{2}$ means that $\mu_{1}$ is absolutely continuous with respect to $\mu_{2}$.

Lemma 5 [1]. Suppose $\mu_{X}$ and $\mu_{Y}$ are two probability measures on $B$ [ß]. Let $\mu_{X} \otimes \mu_{Y}$ denote product measure on $(\Re \times \Re, B[\Re] \times B[\Re])$. The set $\{(x, y)$ : $x+y \in A\}$ belongs to $B[\Re] \times B[\Re]$ for all $A \in B[\Re]$. Define a probability measure $\mu_{X+Y}$ on $B[\Re]$ by $\mu_{X+Y}(A)=\mu_{X} \otimes \mu_{Y}\{(x, y): x+y \in A\}$, and for each fixed $v$ in $\mathscr{B}$, a measure $\mu_{X+v}$ by $\mu_{X+v}(A)=\mu_{X}\{x: x+v \in A\}$. We then bave the following results:

(1) If $\mu_{X+y} \ll \mu_{X}$ a.e. $d \mu_{Y}(y)$, then $\mu_{X+Y} \ll \mu_{X}$.

(2) If $\mu_{X} \ll \mu_{X+y}$ a.e. $d \mu_{Y}(y)$, then $\mu_{X} \ll \mu_{X+Y^{\bullet}}$

(3) If $\mu_{X} \sim \mu_{X+y}$ a.e. $d \mu_{Y}(y)$, then $\mu_{X} \sim \mu_{X+Y^{*}}$

We thus consider Gaussian measures $\mu_{X}$ and the class of measures $\mu_{X+Y}$ defined as in the lemma, where $\mu_{Y}$ need not be Gaussian. An obvious consequence of this lemma is the following. If $B$ is a Hilbert space, so that $\mu_{X}$ has a covariance operator $K$, then $\mu_{X+Y} \sim \mu_{X}$ if $\mu_{Y}\left[\operatorname{range}\left(K^{1 / 2}\right)\right]=1$. For the case where $\mathscr{B}$ is not a Hilbert space, one can imbed $\mathscr{B}$ as a dense measurable linear manifold in a separable Hilbert space $\mathcal{H}$, and work with the covariance operator of the extension of $\mu_{X}$. There are thus obvious extensions of the results of the preceding section. Two such extensions are given below.

Theorem 9. Suppose $\left\{Z_{n}\right\}, n=1,2, \cdots$, and $\left\{V_{n}\right\}, n=1,2, \cdots$, are two families of real random variables defined on a probability space $(\Omega, \beta, P)$. Suppose that $\left\{Z_{n}\right\}$ is Gaussian, and that $\left\{Z_{n}\right\}$ is independent of $\left\{V_{n}\right\}$. Let $K(n, m)$ denote the covariance of $Z_{n}$ and $Z_{m}$, and let $R(n, m)$ denote the correlation of $V_{n}$ and $V_{m}$. Let $\left\{e_{n}\right\}$ be any set of real-valued functions defined and continuous on the interval $T$.

Let $g=\left(g_{1}, g_{2}, \cdots\right)$ be a sequence of real numbers such that $g_{i}>0$, all $i$, $\Sigma g_{i}^{2}<\infty$, and $\Sigma_{i=1}^{\infty} g_{i}^{2} E\left[Z_{i}^{2}(\omega)\right]<\infty$. Suppose that one of the following conditions is satisfied: 
(1) $\left(\Sigma_{i} g_{i} V_{i}(\omega) x_{i}\right)^{2} \leq k(\omega) \Sigma_{i, j} g_{i} g_{j} x_{i} x_{j} K(i, j)$ for a fixed random variable $k$, all $x \in l_{2}$, a.e. $d P(\omega)$.

(2) For some matrix operator $S$ in $l_{2}$ such that $\Sigma_{i} S_{i i}<\infty, R^{\prime}=K_{1}^{1 / 2} S K_{1}^{1 / 2}$, where the matrix operators $R^{\prime}$ and $K_{1}$ are defined by $R^{\prime}(i, j)=g_{i} g_{j} R(i, j)$ and $K_{1}(i, j)=g_{i} g_{j} K(i, j)$.

(3) (a) $\Sigma_{i \notin j}\left(K^{2}(i, j) / K(i, i) K(j, j)\right)<1$ (summation over $i, j$ sucb that $K_{i i} \neq 0$, $\left.K_{j j} \neq 0\right)$ and $R(i, i) \leq k K(i, i)$ for all $i$ and some finite scalar $k$,

(b) $\Sigma_{j}\left(V_{i}^{2}(\omega) / K(i, i)\right)<\infty$ a.e. $d P(\omega)$.

Then Theorem 4 and Theorem 5 bold with $\left\{Z_{n}\right\}$ replaced by $\left\{Z_{n}+V_{n}\right\}$.

Proof. Let $\left(g_{i}\right)$ be defined as in the theorem, and set $Y_{n}(\omega)=g_{n} Z_{n}(\omega)$. Then $Y(\omega) \equiv\left(Y_{1}(\omega), Y_{2}(\omega), \cdots\right)$ is in $l_{2}$ a.e. $d P(\omega)$, and hence induces from $P$ a Gaussian measure $\mu_{Y}$ on $B\left[l_{2}\right] . \mu_{Y}$ has a covariance operator $K_{Y}, K_{Y}(i, j)=$ $g_{i} g_{j} K(i, j)$. Consider condition (1) of the theorem. This condition implies that $V^{\prime}(\omega) \equiv\left(g_{1} V_{1}(\omega), g_{2} V_{2}(\omega), \cdots\right)$ belongs to $l_{2}$ a.e. $d P(\omega)$, and thus $V^{\prime}$ induces from $P$ a probability measure $\mu^{\prime}$ on $B\left[l_{2}\right]$. Moreover, condition $(1)$ is a necessary and sufficient condition that $V^{\prime}(\omega) \epsilon$ range $\left(K_{Y}^{1 / 2}\right)$ for almost all $\omega[5]$. Let $\mu_{1}$ be the probability measure induced on $B\left[l_{2}\right]$ by $Y+V^{\prime}$. Since $\mu^{\prime}\left[\operatorname{range}\left(K_{Y}^{1 / 2}\right)\right]=1$, $\mu_{1} \sim \mu_{Y}$, by Lemma 5 . The assertions of the theorem are now clear when (1) is satisfied. For example, defining $e_{n}^{\prime \prime}(t)=g_{n}^{-1} e_{n}(t)$,

$$
\begin{aligned}
P\left(A^{\prime}\right) & =\mu_{1}\left\{x \in l_{2}: \sum_{1}^{N} x_{n} e_{n}^{\prime \prime}(t) \text { converges uniformly on } T\right\} \\
& =\mu_{Y}\left\{x \in l_{2}: \sum_{1}^{N} x_{n} e_{n}^{\prime \prime}(t) \text { converges uniformly on } T\right\}
\end{aligned}
$$

because the latter quantity is 0 or 1 , by Theorem 5 and $\mu_{1} \sim \mu_{Y}$.

Condition (2) implies condition (1) [1].

Condition (3a) of the theorem is equivalent to

$$
\sum_{i \neq j} \frac{K_{Y}^{2}(i, j)}{K_{Y}(i, i) K_{Y}(j, j)}<1
$$

and $g_{i}^{2} R(i, i) \leq k K_{Y}(i, i)$, all $i$, some finite $k$. The assumption that $g_{i}^{2} R(i, i) \leq$ $k K_{Y}(i, i)$ implies that $V^{\prime}(\omega) \in l_{2}$ a.e. $d P(\omega), V_{i}^{\prime}(\omega)=g_{i} V_{i}(\omega)$. Kuelbs has shown [14] that the first part of (3a) implies that $\mu_{Y}$ and $\mu_{Y}^{\prime}$ are mutually absolutely continuous, where $\mu_{Y}^{\prime}$ is the Gaussian measure on $B\left[l_{2}\right]$ having the same mean element as $\mu_{Y}$, but with diagonal covariance matrix $Q, Q_{i i}=K_{Y}(i, i)$. A necessary condition for mutual absolute continuity of $\mu_{Y}^{\prime}$ and $\mu_{Y}$ is that $Q^{1 / 2}$ and $K_{Y}^{1 / 2}$ have the same range space [1]. Note that $\Sigma_{i}\left(V_{i}^{2}(\omega) / K(i, i)\right)=\Sigma_{i}\left(\left[V_{i}^{\prime}(\omega)\right]^{2} / K_{Y}(i, i)\right)$. 
We now show that if this sum is finite for almost all $\omega$, and if condition ( $3 a$ ) is satisfied, then $V^{\prime}(\omega)$ belongs to range $\left(Q^{1 / 2}\right)$ a.e. $d P(\omega)$. First, $V^{\prime}(\omega)$ belongs almost surely to the closure of the range of $R^{\prime}, R_{i j}^{\prime}=g_{i} g_{j} R(i, j)$. To see this, we note that if $\left\{b_{i}\right\}$ are the complete orthonormal (in $l_{2}$ ) eigenvectors of $R^{\prime}$, and $b_{i}^{\prime}$ are those eigenvectors corresponding to the zero eigenvalues of $R^{\prime}$, then

$$
\int_{\Omega} \sum_{k, j} b_{i k}^{\prime} b_{i j}^{\prime} V_{k}^{\prime}(\omega) V_{j}^{\prime}(\omega) d P(\omega)=0
$$

for each $b_{i}^{\prime}$. Hence, $P\left\{\omega: V^{\prime}(\omega) \perp b_{i}^{\prime}\right\}=1$, each $b_{i}^{\prime}$, so that $P\left\{\omega: V^{\prime}(\omega)\right.$ is not orthogonal to $\left.b_{i}^{\prime}\right\}=0$, all $b_{i}^{\prime}$. Noting that $\left\{\omega: V^{\prime}(\omega) \notin \overline{\left.\operatorname{range}\left(R^{\prime}\right)\right\}}=\bigcup_{b_{i}^{\prime}\left\{\omega: V^{\prime}(\omega)\right.}\right.$ is not orthogonal to $\left.b_{i}^{\prime}\right\}$, we have that $P\left\{\omega: V^{\prime}(\omega) \in \overline{\operatorname{range}\left(R^{\prime}\right)}\right\}=1$. Next we show that range $(Q) \supset$ range $\left(R^{\prime}\right)$. Suppose $Q_{i i}=0$. Then the element $e_{i} \in l_{2}$, $e_{i_{j}}=\delta_{i j}$, is in the null space $\pi_{Q}$ of $Q$, and $\Sigma_{k, j} e_{i_{j}} e_{i_{k}} R^{\prime}(j, k)=R^{\prime}(i, i)=0$, since $R^{\prime}(i, i) \leq k K_{Y}(i, i)$, all $i$, and $Q_{i i}=K_{Y}(i, i)$. Hence $e_{i}$ is in the null space of $R^{\prime}$. The elements $e_{i}$ such that $e_{i}$ is in $\pi_{Q} \operatorname{span} \pi_{Q}$, so that the null space of $Q$ is contained in the null space of $R^{\prime}$. This implies that range( $\left(R^{\prime}\right) C$ $\overline{\text { range }(Q)}$. Hence, $V^{\prime}(\omega) \in \overline{\text { range }(Q)}\left(=\overline{\text { range }\left(Q^{1 / 2}\right)}\right)$ for almost all $\omega$. We now note that if $x \in l_{2}$ and $x \in \overline{\text { range }(Q)}$, then $x \in$ range $\left(Q^{1 / 2}\right)$ if and only if $\Sigma_{i}\left(x_{i}^{2} / Q_{i i}\right)<\infty$. Hence, the assumption that $R(i, i) \leq k K_{Y}(i, i)$, all $i$, and the assumption that $\Sigma_{i}\left(\left[V_{i}^{\prime}(\omega)\right]^{2} / K_{Y}(i, i)\right)<\infty$ a.e. $d P(\omega)$ imply that $V^{\prime}(\omega) \epsilon$ range $\left(Q^{1 / 2}\right)$ a.e. $d P(\omega)$. The assumption that $\Sigma_{i \neq j}\left(K^{2}(i, j) / K(i, i) K(j, j)\right)<1$ implies that range $\left(Q^{1 / 2}\right)=$ range $\left(K_{Y}^{1 / 2}\right)$. Thus the assumptions of $(3)$ imply that $V^{\prime}(\omega) \in \operatorname{range}\left(K_{Y}^{1 / 2}\right)$ a.e. $d P(\omega)$. Hence, assumption (3) implies assumption (1), and the proof is completed.

\section{REFERENCES}

1. C. R. Baker, On equivalence of probability measures, Ann. Probability 1 (1973), 690-698.

2. R. H. Cameron and R. E. Graves, Additive functionals on a space of continuous functions. I, Trans. Amer. Math. Soc. 70 (1951), 160-176. MR 12, 718.

3. R. L. Dobrušin, Properties of sample functions of a stationary Gaussian process, Teor. Verojatnost. i Primenen. 5 (1960), 132-134 = Theor. Probability Appl. 5 (1960), 120-122. MR $25 \# 2644$.

4. J. L. Doob, Stochastic processes, Wiley, New York; Chapman \& Hall, London, 1953. MR 15, 445.

5. R. G. Douglas, On majorization, factorization, and range inclusion of operators in Hilbert space, Proc. Amer. Math. Soc. 17 (1966), 413-415. MR 34 \#3315.

6. A. M. Garsia, E. R. Rodemich and H. Rumsey, Jro, A real variable lemma and the continuity of paths of some Gaussian processes, Indiana Univ. Math. J. 20 (1970/71), 565-578. MR $42 \# 2534$.

7. I. M. Gel 'fand and A. M. Yaglom, Calculation of the amount of information about a random function contained in another such function, Uspehi Mat. Nauk 12 (1957), no. 1 (73), 3-52; English transl., Amer. Math. Soc. Transl. (2) 12 (1959), 199-246. MR 18, 980; MR 22 \#4574a. 
8. J. Hájek, On linear statistical problems in stochastic processes, Czechoslovak Math J. 12 (1962), 404-444. MR 27 \#2070.

9. K. Itô and M. Nisio, On the oscillation functions of Gaussian processes, Math. Scand. 22 (1968), 209-223. MR 39 \#4918.

10. N. C. Jain and G. Kallianpur, A note on uniform convergence of stochastic processes, Ann. Math. Statist. 41 (1970), 1360-1362. MR 42 \#6931.

11. N. C. Jain, A zeroone law for Gaussian processes, Proc. Amer. Math. Soc. 29 (1971), 585-587. MR 43 \#4099.

12. G. Kallianpur, Zero-one laws for Gaussian processes, Trans. Amer. Math. Soc. 149 (1970), 199-211. MR $42 \# 1200$.

13. E. J. Kelly, I. S. Reed and W. L. Root, The detection of radar echoes in noise. I, SIAM J. Appl. Math. 8 (1960), 309-341.

14. J. Kuelbs, Gaussian measures on a Banach space, J. Functional Analysis 5 (1970), 354-367. MR 41 \#4639.

15. H. J. Landau and L. A. Shepp, On the supremum of a Gaussian process, Sankhyā Ser. A 32 (1970), 369-378.

16. E. Mourier, Éléments aléatoires dans un espace de Banach, Ann. Inst. H. Poincaré 13 (1953), 161-244. MR 16, 268.

17. K. R. Parathasarathy, Probability measures on metric spaces, Probability and Math. Statist., no. 3, Academic Press, New York, 1967. Chapter 1, §3. MR 37 \#2271.

18. T. S. Pitcher, On the sample functions of processes which can be added to a Gaussian process, Ann. Math. Statist. 34 (1963), 329-333. MR 26 \#4405.

19. Ju. V. Rozanov, Infinite-dimensional Gaussian distributions, Trudy Mat. Inst. Steklov. 108 (1968) = Proc. Steklov In st. Math. 108 (1968).

20. N. N. Vakhania, Sur une propriété des répartitions normales des probabilités dans les espaces $l_{p}(1 \leq p<\infty)$, et $H$, C. R. Acad. Sci. Paris 260 (1965), 1334-1336. MR $30 \# 4282$.

21. - Sur les répartitions de probabilités dans les espaces de suites numériques, C. R. Acad. Sci. Paris 260 (1965), 1560-1562. MR 30 \#4283.

DEPARTMENT OF STATISTICS, UNIVERSITY OF NORTH CAROLINA, CHAPEL HILL, NORTH CAROLINA 27514 